Pacific Journal of Mathematics

ON A NEW METHOD FOR DEFINING THE NORM OF 


\title{
ON A NEW METHOD FOR DEFINING THE NORM OF FOURIER-STIELTJES ALGEBRAS
}

\author{
MARTIN E. WALter \\ This paper is dedicated to the memory of Henry Abel Dye
}

\begin{abstract}
P. Eymard equipped $B(G)$, the Fourier-Stieltjes algebra of a locally compact group $G$, with a norm by considering it the dual Banach space of bounded linear functionals on another Banach space, namely the universal $C^{*}$-algebra, $C^{*}(G)$. We show that $B(G)$ can be given the exact same norm if it is considered as a Banach subalgebra of $\mathscr{D}\left(C^{*}(G)\right)$, the Banach algebra of completely bounded maps of $C^{*}(G)$ into itself equipped with the completely bounded norm. We show here how the latter approach leads to a duality theory for finite (and, more generally, discrete) groups which is not available if one restricts attention to the "linear functional" [as opposed to the "completely bounded map"] approach.
\end{abstract}

1. Preliminaries. For most of this paper $G$ will be a finite (discrete) group, although we consider countably infinite discrete groups in the last section. We now summarize certain aspects of duality theory that we will need. The discussion is for a general locally compact group $G$ whenever no simplification is achieved by assuming $G$ to be finite. The reader familiar with Fourier and Fourier-Stieltjes algebras may proceed to $\S 2$ and refer back to this section as might be necessary.

From [3] we see that the original motivation for the investigation of Fourier-Stieltjes algebras came from the measure algebra, $M^{1}(\hat{G})$, where $\hat{G}$ is the (Pontryagin-van Kampen) dual group of locally compact abelian group $G$ and $M^{1}(\hat{G})$ is the $*$-Banach convolution algebra of (finite) complex regular Borel measures on $\hat{G}$. In $[5, \S 24]$ there is a thorough discussion of $\hat{G}$ (where $G$ is abelian) and the accompanying Pontryagin-van Kampen duality theory.

Digressing briefly we note that in $[5, \S 19.12]$ the convolution algebra $M(G)$, in the notation of Hewitt and Ross, is defined; and it is studied in [5, §19]. (Here $G$ is a not necessarily abelian, locally compact group.)

We use Dixmier's notation $M^{1}(G)$, cf., [2, §13.22], instead of Hewitt and Ross' $M(G)$, though they are identical mathematical objects. We recommend $[2, \S 13]$ for a study of $M^{1}(G)$ as it relates to the set of 
continuous, positive definite functions on $G$, labeled $P(G)$, and to the continuous unitary representations of $G$ on Hilbert space.

We note for emphasis that as a Banach space $M^{1}(G)$ is isometrically isomorphic to the dual space of bounded, linear functionals on $C_{0}(G)$, the continuous, complex-valued functions that vanish at infinity. This duality, written in Bourbaki notation, is

$$
\langle f, \mu\rangle=\int_{G} f(x) d \mu(x),
$$

where

$$
\mu \in M^{1}(G) \text { and } f \in C_{0}(G) .
$$

The formula for convolution is

$$
\begin{gathered}
\langle f, \mu * \nu\rangle=\int_{G \times G} f(x y) d \mu(x) d \nu(y), \\
f \in C_{0}(G), \quad \mu, \nu \in M^{1}(G) .
\end{gathered}
$$

The formula for the involution on $M^{1}(G)$ is

$$
\left\langle f, \mu^{*}\right\rangle=\int_{G} f(x) d \mu^{*}(x)=\overline{\int \overline{f\left(x^{-1}\right)} d \mu(x)},
$$

$f \in C_{0}(G), \mu \in M^{1}(G)$, overbars denoting complex-conjugation. Finally, the norm of $\mu \in M^{1}(G)$ is given by

$$
\|\mu\|=\sup \left\{|\langle f, \mu\rangle|:\|f\|_{\infty} \leq 1, f \in C_{0}(G)\right\}
$$

where

$$
\|f\|_{\infty}=\sup \{|f(x)|: x \in G\}
$$

Returning to the original motivation for the investigation of the Fourier-Stieltjes algebra, denoted $B(G)$, for a group $G$ we note that $B(G)$ as an algebra is just the (inverse) Fourier-Stieltjes transform, cf., $[5, \S 23.9]$ or $[9, \S 1.3 .3]$, of $M^{1}(\hat{G})$. By Bochner's Theorem, [9, $\left.§ 1.4 .3\right]$, since every $\mu \in M^{1}(\hat{G})$ is a linear combination of at most 4 ("pointwise") non-negative measures in $M^{1}(\hat{G})$ (by Jordan's decomposition theorem, [9, p. 266]), every element $b \in B(G)$ is a linear combination of at most 4 continuous positive definite functions on $G$. If we equip $B(G)$ with the norm it naturally inherits from $M^{1}(\hat{G})$ [more precisely, if $\hat{\mu} \in B(G)$ is the (inverse) Fourier-Stieltjes transform of $\mu \in M^{1}(G)$, then $\|\hat{\mu}\|_{B(G)} \stackrel{\text { (def.) }}{\equiv}\|\mu\|_{M^{\prime}(G)}$, then $B(G)$ is a commutative, Banach algebra with addition and multiplication of elements defined pointwise, e.g., $\left(b_{1} b_{2}\right)(x)=b_{1}(x) b_{2}(x), b_{1}, b_{2} \in B(G), x \in G$. 
P. Eymard's great insight was that virtually this same Banach algebra could be defined for nonabelian, locally compact groups $G$. We very briefly sketch Eymard's construction. We start with $L^{1}(G)$, the 2sided *-ideal in $M^{1}(G)$, consisting of measures absolutely continuous with respect to (left) Haar measure on $G$, cf. [2, §13.2]. We let $C^{*}(G)$ denote the universal enveloping $C^{*}$-algebra of $L^{1}(G)$, cf. [2, §13.9]. We note that if $G$ were abelian, then $C^{*}(G)$ identifies with $C_{0}(\hat{G})$ and the natural *-epimorphism (also surjection for finite $G$ ), $L^{1}(G) \rightarrow C^{*}(G)$, identifies with the Fourier transform. We then have that $P(G)$ identifies with the positive forms on $C^{*}(G)$, viz. $C^{*}(G)_{+}^{\prime}$, cf. [2, §2.7.5]. Now $P(G)$ is a semigroup with multiplication defined pointwise on $G$, i.e., if $p_{1}, p_{2} \in P(G)$, then $p_{1} p_{2} \in P(G)$ where $\left(p_{1} p_{2}\right)(x)=p_{1}(x) p_{2}(x)$ for all $x \in G$. Thus the finite linear combinations of $P(G)$ form an algebra, called $B(G)$, the Fourier-Stieltjes algebra of $G$. But identifying $P(G)$ with $C^{*}(G)_{+}^{\prime}$, the finite linear combinations of continuous positive functionals form a Banach space, namely $C^{*}(G)^{\prime}$, the Banach space of bounded linear functionals on $C^{*}(G)$. A seminal result is thus:

EyMARD's TheOREM. The Fourier-Stieltjes algebra, $B(G)$ is a commutative Banach algebra given the norm it inherits upon identification with $C^{*}(G)^{\prime}$ and the algebra operations of pointwise sum and product it inherits when identified with the linear combinations of continuous positive definite functions on $G$.

We observe that Eymard also defined the Fourier algebra of $G, A(G)$, to be the closure in $B(G)$ of the subalgebra of functions in $B(G)$ with compact support. Thus $A(G)$ is a 2 -sided ideal in $B(G)$. When $G$ is abelian, it follows from Wiener's Tauberian theorem that $A(G)$ is the (inverse) Fourier transform of $L^{1}(\hat{G})$. Also, $A(G)=B(G)$ if and only if $G$ is compact.

We note in passing that $B(G)$ and $A(G)$ are complete invariants of $G$, even for nonabelian $G$ and that a duality theory analogous to Pontryagin duality is possible, cf., [11], [12].

In [13] (Theorem 1, p. 84), we observed that $B(G)$ arises in a somewhat different context than that of Eymard's Theorem above. Namely, let $\mathscr{D}\left(C^{*}(G)\right)$ be the Banach algebra of completely bounded maps of $C^{*}(G)$ into $C^{*}(G)$ equipped with the completely bounded norm. For a discussion of completely positive maps see [10], [7]. See [7] for a discussion of completely bounded maps. 
Now let $p$ be a continuous, positive definite function on locally compact group $G$. We can define a map $T_{p}$ of $L^{1}(G)$ into $L^{1}(G)$ as follows:

$$
T_{p}: f \in L^{1}(G) \mapsto p f \in L^{1}(G)
$$

where $p f$ is the pointwise product on $G$. The map $T_{p}$ extends to a map of $C^{*}(G)$ into $C^{*}(G)$ which is completely positive (hence completely bounded). The completely bounded norm of $T_{p}$ is $p(e)$, the value of $p$ at the identity $e$ of $G$; and this is also the norm of $p$ when it is identified with a positive linear functional on $C^{*}(G)$. If $b \in$ $B(G), T_{b} \in \mathscr{D}\left(C^{*}(G)\right)$ is also defined via pointwise multiplication; and as [13, Theorem 1] states (in part), the completely bounded norm of $T_{b}$, denoted $\left\|T_{b}\right\|_{\mathscr{D}}$, is the same as the norm of $b$ in $B(G)$ when $b$ is viewed as a linear functional on $C^{*}(G)$. Thus $B(G)$ may not only be viewed as the dual space of linear functionals on $C^{*}(G)$, it naturally arises as a commutative, Banach algebra of completely bounded maps of $C^{*}(G)$ into $C^{*}(G)$, i.e., $B(G) \subset \mathscr{D}\left(C^{*}(G)\right)$.

It is this observation, namely that $B(G)$ can be viewed as a "naturally occurring" subalgebra of $\mathscr{D}\left(C^{*}(G)\right)$, which we now wish to apply to the theory of finite (and eventually to countably discrete) groups. Before proceeding to the main point of this article, however, we need to note some final results from [13], [4] and [8].

First, let $\Gamma_{n}=\left\{e_{i j}: 1 \leq i, j \leq n\right\}$, the matrix units for $M_{n}$, the $n \times n$ complex matrices. Thus $e_{i j}$ is the $n \times n$ matrix with a single 1 in the spot determined by the intersection of the $i$ th row and $j$ th column with zeros everywhere else. In [13] $\Gamma_{n}$, more abstractly known as the principal transitive groupoid on $n$-elements, is studied; and a duality theory much analogous to that for groups is developed. In particular, there is a slightly larger variety of algebras (with their accompanying dual algebras) to choose from than there is in the group case. In the case of a group $G, L^{1}(G)$ and $A(G)$ are "dual" to each other, and $M^{1}(G)$ and $B(G)$ are "dual". In the case of groupoid $\Gamma_{n}$ we can define $L^{1}\left(\Gamma_{n}\right)$ to be $\left(M_{n}, *,\|\cdot\|_{L^{1}}\right)$, namely, $M_{n}$ with the matrix product, $*$, and norm $\left\|\left(a_{i j}\right)\right\|_{L^{1}}=\sum_{i, j=1}^{n}\left|a_{i j}\right|$. This $L^{1}\left(\Gamma_{n}\right)$ is a $*$-Banach algebra; it is also the dual space of $L^{\infty}\left(\Gamma_{n}\right)$. If one had to pick a dual algebra for $L^{1}\left(\Gamma_{n}\right)$ one would be led to $\left(M_{n}, \circ,\|\cdot\|_{\mathrm{Tr}}\right)$, i.e., $M_{n}$ with Schur-Hadamard product and trace norm. Here the Schur product is defined by $\left(a_{i j}\right) \circ$ $\left(b_{i j}\right)=\left(a_{i j} b_{i j}\right)$, where $\left(a_{i j}\right)$ and $\left(b_{i j}\right)$ are in $M_{n}$. Also $\left\|\left(a_{i j}\right)\right\|_{\mathrm{Tr}}=$ $\operatorname{Tr}\left(\left|\left(a_{i j}\right)\right|\right)=\operatorname{Tr}\left[\left(a_{i j}\right)^{*}\left(a_{i j}\right)\right]^{1 / 2}$, where $\left(a_{i j}\right)^{*}=\left(\overline{a_{i j}}\right)$ and $\operatorname{Tr}$ is the "sum of the diagonal elements". By [13, Proposition 5] $\left(M_{n}, \circ,\|\cdot\|_{\mathrm{Tr}}\right)$ is a 
commutative algebra and it is the dual space of $\left(M_{n}, *,\|\cdot\|\right)$ where $\|\cdot\|$ denotes the usual operator-norm or $C^{*}$-norm.

It might seem that the pair of algebras $L^{1}\left(\Gamma_{n}\right)=\left(M_{n}, *,\|\cdot\|_{L^{1}}\right)$ and $\left(M_{n}, \circ,\|\cdot\|_{\mathrm{Tr}}\right)$ would be the natural candidates as analogues for the pair $L^{1}(G), A(G)$ where $G$ is a group. This, of course, is one possibility. There are, however, some difficulties with this approach. If we allow $n$ to be infinite or if we look at more general groupoids, the "obvious" candidate spaces fail to be Banach algebras with the dual space norm. The dual space norm appears to be "too large", in general, especially for groupoids with an infinite number of units.

We do have an alternative, however. If we take the $C^{*}$-completions of $L^{1}\left(\Gamma_{n}\right)$ and $\left(M_{n}, \circ,\|\cdot\|_{\mathrm{Tr}}\right)$, we get $\left(M_{n}, *,\|\cdot\|\right)$ and $\left(M_{n}, \circ,\|\cdot\|_{\infty}\right)$, respectively, where $\left\|\left(a_{i j}\right)\right\|_{\infty}=\sup \left\{\left|a_{i j}\right|: 1 \leq i, j \leq n\right\}$. We define $C^{*}\left(\Gamma_{n}\right) \equiv\left(M_{n}, *,\|\cdot\|\right)$, viz., $M_{n}$ with its usual $C^{*}$-algebra structure; and we define $C^{*}\left(\hat{\Gamma}_{n}\right) \equiv C\left(\Gamma_{n}\right)=\left(M_{n}, \circ,\|\cdot\|_{\infty}\right)$, where $C\left(\Gamma_{n}\right)$ is the set of all (continuous) complex-valued functions on the (discrete) space $\Gamma_{n}$. Then there arise in $\mathscr{D}\left(C^{*}\left(\Gamma_{n}\right)\right)$ and $\mathscr{D}\left(C^{*}\left(\hat{\Gamma}_{n}\right)\right)$ analogues of $A(G)$ and $L^{1}(G)$, respectively, see $[13, \S 3]$.

The analogues of $L^{1}(G)$ we will denote by $L_{r}^{1}\left(\Gamma_{n}\right)$ and $L_{l}^{1}\left(\Gamma_{n}\right)$, where $r$ denotes the fact that $L_{r}^{1}\left(\Gamma_{n}\right)$ arises as an algebra of operators in $\mathscr{D}\left(C^{*}\left(\hat{\Gamma}_{n}\right)\right)$ that "act on the right" and $L_{l}^{1}\left(\Gamma_{n}\right)$ "acts on the left". Thus we define $L_{r}^{1}\left(\Gamma_{n}\right) \equiv\left(M_{n}, *,\|\cdot\|_{I, r}\right)$ where $\left\|\left(a_{i j}\right)\right\|_{I, r}=\sup _{i} \sum_{j}\left|a_{i j}\right|$. We define $L_{l}^{1}\left(\Gamma_{n}\right) \equiv\left(M_{n}, *,\|\cdot\|_{I, d}\right)$ where $\left\|\left(a_{i j}\right)\right\|_{I, d}=\sup _{j} \sum_{i}\left|a_{i j}\right|$. We use the subscript $d$, following previous authors, though the subscript $l$ might seem more natural in this setting. The important point, which as far as we know was unnoticed before [13], is that these norms arise naturally as completely bounded norms.

Now the natural (cf., Theorem 1 below) dual of $L_{r}^{1}\left(\Gamma_{n}\right)$ (and/or $\left.L_{l}^{1}\left(\Gamma_{n}\right)\right)$ is $A\left(\Gamma_{n}\right)$ which we define to be the subalgebra of $\mathscr{D}\left(C^{*}\left(\Gamma_{n}\right)\right)$ obtained by letting $M_{n}$ act on $C^{*}\left(\Gamma_{n}\right)$ via the Schur-Hadamard product, i.e., $A\left(\Gamma_{n}\right) \equiv\left(M_{n}, \circ,\|\cdot\|_{\mathscr{D}_{s}}\right)$ where $\left\|\left(a_{i j}\right)\right\|_{\mathscr{D}_{s}}$ is the completely bounded norm of the mapping

$$
\left(x_{i j}\right) \in C^{*}\left(\Gamma_{n}\right) \mapsto\left(a_{i j}\right) \circ\left(x_{i j}\right) \in C^{*}\left(\Gamma_{r}\right) .
$$

The subscript $\mathscr{D}_{s}$ denotes the completely bounded, i.e., "dual", norm arising from the Schur-Hadamard "action".

Now this norm would be intractable if it were not for a "famous" but unpublished (as far as we know) theorem of Uffe Haagerup. In fact, this article rests quite heavily on the following theorem of Haagerup. 
[We note in passing that it follows from Haagerup's work that

$$
\left\|\left(a_{i j}\right)\right\|_{\mathscr{D}_{s}}=\left\|\left(a_{i j}\right)\right\|_{\mathscr{L}\left(C^{*}\left(\Gamma_{n}\right)\right)}=\sup \left\{\left\|\left(a_{i j}\right) \circ\left(x_{i j}\right)\right\|:\left\|\left(x_{i j}\right)\right\| \leq 1\right\},
$$

i.e., the completely bounded norm of the map $\left(x_{i j}\right) \mapsto\left(a_{i j}\right) \circ\left(x_{i j}\right)$ coincides with its bounded norm.]

THEOREM (Haagerup). Let $(\Omega, \mu)$ be a $\sigma$-finite measure space, and let $A$ be the maximal abelian von Neumann algebra $L^{\infty}(\Omega, \mu)$ acting as multiplication operators on $H=L^{2}(\Omega, \mu)$. Let $\phi \in L^{\infty}(\Omega \times \Omega, \mu \times \mu)$ and let $M_{\phi}$ be the operator on $\mathscr{H S}(H)$, the Hilbert-Schmidt operators on $H$, defined by $M_{\phi}\left(Q_{k}\right)=Q_{\phi k}, k \in L^{2}(\Omega \times \Omega, \mu \times \mu)$, where $\left(Q_{k} f\right)(x)=$ $\int_{\Omega} k(s, t) f(t) d \mu(t)$. Then the following are equivalent:

(1) $\left\|M_{\phi} x\right\| \leq\|x\|$ for all $x \in \mathscr{H S S}(H)$.

(2) $M_{\phi}$ has a $\sigma$-weakly continuous extension $\tilde{M}_{\phi}$ to $\mathscr{B}(H)$ such that $\left\|\tilde{M}_{\phi}\right\| \leq 1$. Here $\mathscr{B}(H)$ denotes all bounded, linear operators on $H$.

(3) There exist two sequences of functions $\left(f_{n}\right)_{N \in N}$ and $\left(g_{n}\right)_{n \in N}$ in $L^{\infty}(\Omega, \mu)$ such that

$$
\sum_{n=1}^{\infty}\left|f_{n}(s)\right|^{2} \leq 1 \quad \text { and } \quad \sum_{n=1}^{\infty}\left|g_{n}(s)\right|^{2} \leq 1 \quad \mu \text {-a.e. }
$$

and $\phi(s, t)=\sum_{n=1}^{\infty} f_{n}(s) g_{n}(t) \mu \times \mu$-a.e.

(4) There exist a separable Hilbert space $\mathscr{H}$ and two functions $\xi, \eta \in$ $L^{\infty}(\Omega, \mathscr{H}, \mu)$ such that $\|\xi\|_{\infty} \leq 1,\|\eta\|_{\infty} \leq 1$ and

$$
\phi(s, t)=(\xi(s) \mid \eta(t)) \quad \mu \times \mu \text {-a.e. }
$$

Moreover, any $\sigma$-weakly continuous, $A$ bimodule map on $\mathscr{B}(H)$ with norm less than or equal to 1 is of the form $\tilde{M}_{\phi}$ for a $\phi \in L^{\infty}(\Omega \times \Omega, \mu \times \mu)$ satisfying conditions (1) through (4).

As an immediate consequence of this theorem, we have the following:

COROLlary. Let $M_{n}$ be the $C^{*}$-algebra of complex $n \times n$ matrices. Let

$$
M_{x}: y \in M_{n} \mapsto x \circ y \in M_{n} .
$$

Then $\left\|M_{x}\right\|_{\mathscr{D}_{s}} \leq 1$ if and only if there exists a Hilbert space $\mathscr{H}$ and $2 n$ vectors $\xi_{1}, \ldots, \xi_{n}, \eta_{1}, \ldots, \eta_{n}$ in the unit ball of $\mathscr{H}$ such that

$$
x_{i j}=\left(\xi_{i} \mid \eta_{j}\right), \quad i, j=1, \ldots, n .
$$

A short proof of this corollary is to be found in [8]. 
2. The Cayley representations. In this section we look at an old mathematical construction from a slightly new perspective. Let $G$ be a finite group, and let $g_{1}, g_{2}, \ldots, g_{n}$ be a list of the elements of $G$. Now construct an $n \times n$ array consisting of elements of $G$ :

\begin{tabular}{llllll} 
& $g_{1}$ & $g_{2}$ & $g_{3}$ & & $g_{n}$ \\
\hline$g_{1}^{-1}$ & $e$ & $g_{1}^{-1} g_{2}$ & $g_{1}^{-1} g_{3}$ & $\ldots$ & $g_{1}^{-1} g_{n}$ \\
$g_{2}^{-1}$ & $g_{2}^{-1} g_{1}$ & $e$ & $g_{2}^{-1} g_{3}$ & $\ldots$ & $g_{2}^{-1} g_{n}$ \\
$g_{3}^{-1}$ & $g_{3}^{-1} g_{1}$ & $g_{3}^{-1} g_{2}$ & $e$ & $\ldots$ & $g_{3}^{-1} g_{n}$ \\
$\vdots$ & $\vdots$ & & & & $\vdots$ \\
$g_{n}^{-1}$ & $g_{n}^{-1} g_{1}$ & $\ldots$ & $\ldots$ & $\ldots$ & $e$
\end{tabular}

where the element in the intersection of the $i$ th row and $j$ th column is $g_{i}^{-1} g_{j}$. This array is essentially the multiplication table of $G$ written in a manner that has a certain symmetry. Now one can think of this array as a single element in $C^{*}(G) \otimes M_{n}$, i.e., the $n \times n$ matrices with entries from $C^{*}(G)$, cf. [10, p. 192]. Recall that $C^{*}(G)$ is the (universal) $C^{*}$-completion of the Banach algebra, with usual product and ${ }^{*}$,

$$
L^{1}(G)=\left\{\sum_{i=1}^{n} \lambda_{i} g_{i}: \lambda_{i} \in \mathbf{C}, g_{i} \in G, 1 \leq i \leq n\right\}
$$

where

$$
\left\|\sum_{i=1}^{n} \lambda_{i} g_{i}\right\|_{L^{1}(G)}=\sum_{i=1}^{n}\left|\lambda_{i}\right|
$$

Fortunately, $C^{*}(G)$ can be realized, simply as the left regular representation of $L^{1}(G)$ on the Hilbert space

$$
L^{2}(G)=\left\{\sum_{i=1}^{n} \lambda_{i} g_{i}: \lambda_{i} \in \mathbf{C}, g_{i} \in \mathbf{C}, 1 \leq i \leq n\right\}
$$

where

$$
\left\|\sum_{i=1}^{n} \lambda_{i} g_{i}\right\|_{L^{2}(G)}=\left(\sum_{i=1}^{n}\left|\lambda_{i}\right|^{2}\right)^{1 / 2} .
$$

More precisely, consider $\lambda(f) \xi \stackrel{\text { (def.) }}{\equiv} f * \xi$ for $\xi \in L^{2}(G), f \in L^{1}(G)$, where $f * \xi(x)=\sum_{y \in G} f(y) \xi\left(y^{-1} x\right), x \in G$. This left-regular representation, $\lambda: L^{1}(G) \rightarrow \mathscr{B}\left(L^{2}(G)\right)$ maps $L^{1}(G)$ faithfully (that is $\lambda(f)=$ 0 if and only if $f=0$ ) onto a $C^{*}$-subalgebra of $\mathscr{B}\left(L^{2}(G)\right), \lambda\left(L^{1}(G)\right)$, 
and we have that $\lambda\left(L^{1}(G)\right)$ is isometrically isomorphic to $C^{*}(G)$. Recall that $\left(\sum_{i=1}^{n} \lambda_{i} g_{i}\right)^{*}=\sum_{i=1}^{n} \bar{\lambda}_{i} g_{i}^{-1}$ and that $\lambda\left(f^{*}\right)=\lambda(f)^{*}$ for all $f$ in $L^{1}(G)$.

We can realize an $n \times n$ "multiplication table matrix" $\left(g_{i}^{-1} g_{j}\right)$ as an operator

$$
P=\sum_{i, j=1}^{n} g_{i}^{-1} g_{j} \otimes e_{i j} \in C^{*}(G) \otimes M_{n}
$$

Now

$$
P=\left(\sum_{i=1}^{n} g_{i} \otimes e_{1 i}\right)^{*}\left(\sum_{j=1}^{n} g_{j} \otimes e_{1 j}\right) \geq 0 .
$$

Also $P^{*}=P=P^{2} / n$, i.e., $P / n$ is a projection operator in $C^{*}(G) \otimes M_{n}$. We have the following proposition.

Proposition 1. A "multiplication table projection" of $G$, viz.,

$$
\frac{P}{n}=\frac{1}{n} \sum_{i, j=1}^{n} g_{i}^{-1} g_{j} \otimes e_{i j},
$$

is in fact a self-adjoint (positive), idempotent in $C^{*}(G) \otimes M_{n}$.

If $f: G \rightarrow \mathbf{C}$ is any complex-valued function on finite group $G$, then $f$ is an element of $A(G)$ where we can identify $A(G)$ with either the dual space of $C^{*}(G), C^{*}(G)^{\prime}$, or a certain subalgebra of $\mathscr{D}\left(C^{*}(G)\right)$. More importantly at this moment, however, we can identify $f$ with a certain $n \times n$ matrix via what we shall refer to as a local Cayley representation. Thus we have the following

Definition. Given $G=\left\{g_{1}, g_{2}, \ldots, g_{n}\right\}$ and $f: G \rightarrow \mathbf{C}$, we define a local Cayley representation of $f$ to be that complex matrix, denoted by $\left(f_{i j}\right)$ where $f_{i j}=f\left(g_{i}^{-1} g_{j}\right), i, j=1, \ldots, n$.

REMARK. Note that we refer to "a" local Cayley representation since the representation depends on the specific way in which the elements of $G$ are enumerated.

REMARK. If we consider $f$ (by uniquely "extending") as a linear functional on $C^{*}(G)$ we can obtain a local Cayley representation of $f$ as follows. Let $P / n$ be the "multiplication table projection" of Proposition 1 . Now $P \in C^{*}(G) \otimes M_{n}$, and we can apply the map $f \otimes I_{n}$ to $P$. The result will be the corresponding local Cayley representation of $f$ in $\mathbf{C} \otimes M_{n} \cong M_{n}(\mathbf{C})$. 
REMARK. Clearly $f \in P(G)$ if and only if any one of its local Cayley representations is a positive definite matrix.

The local Cayley representation defines a map, called a local Cayley map determined by $G$,

$$
I_{G}: A(G) \rightarrow\left(M_{n}, \circ,\|\cdot\|_{\mathscr{D}_{s}}\right) \equiv A\left(\Gamma_{n}\right)
$$

which is clearly an algebraic isomorphism (into), cf. Theorem 1 below.

We now define a global Cayley representation of $C^{*}(G)$. Let $\delta_{g_{1}}$ be that function on $G$ which is 1 on $g_{i}$ and 0 elsewhere. Then $I_{G}\left(\delta_{g_{i}}\right)$ is an $n \times n$ permutation matrix, and we leave it to the reader to verify that we have just reconstructed the classical Cayley representation of $G$ into the $n \times n$ permutation matrices. We can extend this representation uniquely to all of $C^{*}(G)$ and we call this unique extension, $I^{G}$, a global Cayley representation. Thus

$$
I^{G}: C^{*}(G) \rightarrow M_{n}
$$

is a *-isomorphism (isometric) of $C^{*}(G)$ into $M_{n}$, the $C^{*}$-algebra of $n \times n$ matrices. Clearly the initial enumeration of the elements of $G$ can affect this map, hence we use again the indefinite article " $a$ ".

We digress briefly from the main thrust of this paper to give a characterization of finite-group $C^{*}$-algebras in terms of an "index" defined on all finite dimensional $C^{*}$-algebras.

Definition 2. If $A$ is a finite dimensional $C^{*}$-algebra we define $\gamma(A)$ to be the order of a group of unitary elements of $A$ with the following two properties:

(i) This group of unitary elements of $A$ span $A$, i.e., every element of $A$ is a linear combination of elements of this group, and

(ii) no group of unitary elements of $A$ of strictly lesser order spans A.

REMARKs. Every finite dimensional $C^{*}$-algebra $A$ has a unit; thus it makes sense to speak of the unitary elements of $A$. We leave it to the reader to show that $\gamma(A)$ is well-defined. Though $\gamma(A)$ is welldefined there may be two non-isomorphic groups of unitary elements of $A$ which yield the index $\gamma(A)$. A classical example is $A=\mathrm{C}^{4} \otimes M_{2}$ which is spanned by the eight element quaterion group and the eight element dihedral group. The letter $\gamma$ was chosen to stand for "the Gasemyr index" since this index was discovered by the author in a conversation with Jørund Gasemyr.

Various properties of $\gamma$ can be shown, but we only prove the following. 
Proposition 2. A finite dimensional $C^{*}$-algebra $A$ is *-isomorphic to the group $C^{*}$-algebra of a finite group if and only if $\gamma(A)=\operatorname{dim} A$. In this case $A \cong C^{*}(G)$ for any group $G$ of unitary elements of $A$ which form a basis of $A$ as a vector space.

Proof. Clearly $\gamma(A) \geq \operatorname{dim} A$, always. If $A$ is the group $C^{*}$-algebra of a finite group $G$, then it is well known (and easy to see) that there is a group of unitary elements of $A$ isomorphic with $G$ such that this group is a basis of $A$ as a vector space. Thus $\gamma(A)=\operatorname{dim} A$. Conversely, suppose $\gamma(A)=\operatorname{dim} A$. Then there is a group of unitary elements, $G$, in $A$ which is a basis for $A$. Consider the universal enveloping $C^{*}$ algebra of $G, C^{*}(G)$. By universality there is a $*$-homomorphism of $C^{*}(G)$ onto $A$. But $G$ is a basis of $A$ and of $C^{*}(G)$, thus $C^{*}(G) \cong A$.

The following result is some of our strongest evidence that the "completely bounded operator norm" on $A\left(\Gamma_{n}\right)$ is the "correct" norm for duality studies.

THEOREM 1. If $G$ is a group of order $n$ and $A(G)$ is its Fourier algebra, then the local Cayley map $I_{G}: A(G) \rightarrow A\left(\Gamma_{n}\right)$ is an isometric isomorphism strictly into for $n>1$. The analogous map, $A(G) \rightarrow$ $\left(M_{n}, \circ,\|\cdot\|_{\mathrm{Tr}}\right)$, is not isometric unless $n=1$.

REMARK. Thus given the choice of equipping the dual of groupoid $\Gamma_{n}$ with the "dual space norm" $\|\cdot\|_{\mathrm{Tr}}$ or the "completely bounded operator norm" $\|\cdot\|_{\mathscr{D}_{s}}$, we see that only the latter is compatible with the norm on the Fourier algebra offor $n>1$. finite group $G$ of order $n$. In this latter case then $A(G) \subset A\left(\Gamma_{n}\right)$, where $A(G)$ is isometrically a subalgebra of $A\left(\Gamma_{n}\right)$.

Proof of Theorem 1. Let $a \in A(G)$. By [3, $\$ 2.14$ and Chapitre 3] there are $\xi$ and $\eta$ in $L^{2}(G)$ such that $a=\xi * \tilde{\eta}$ and $\|a\|_{A(G)}=$ $\|\xi\|_{L^{2}(G)}\|\eta\|_{L^{2}(G)}$. Note: $\tilde{\eta}(g)=\overline{\eta\left(g^{-1}\right)}$ for $g$ in $G$. Without loss of generality suppose that $\|a\|_{A(G)}=1=\|\xi\|_{L^{2}(G)}=\|\eta\|_{L^{2}(G)}$. Now by means of a fixed local Cayley representation we can identify $a, \xi$ and $\eta$ with certain $n \times n$ matrices denoted $\left(a_{i j}\right),\left(\xi_{i j}\right),\left(\eta_{i j}\right)$, respectively. Let us look closely at these matrices. If we assume $g_{1}=e$ is the group identity, then the first row of $\left(\xi_{i j}\right)$ is $\xi\left(g_{j}\right), j=1,2, \ldots, n$, i.e. $\xi$. The $i$ th row is $\xi\left(g_{i}^{-1} g_{j}\right) j=1,2, \ldots, n$, or $\lambda\left(g_{i}\right) \xi$. Similarly for $\eta$. Now referring to the notation of the corollary to Haagerup's theorem we have $2 n$ vectors, $\xi_{1}, \ldots, \xi_{n}, \eta_{1}, \ldots, \eta_{n}$ ("two cross-sections of a Hilbert 
bundle on the units of $\Gamma_{n}$ ") where $\xi_{i}=\lambda\left(g_{i}\right) \xi$ and $\eta_{j}=\lambda\left(g_{j}\right) \eta, i, j=$ $1, \ldots, n$. Now $\left\|\xi_{i}\right\|_{L^{2}(G)}=1=\left\|\eta_{j}\right\|_{L^{2}(G)}$ for all $i, j$. Now we claim that since $a=\xi * \tilde{\eta}$ on $G$, then for all $i, j, a_{i j}=\left(\xi_{i} \mid \eta_{j}\right)$, i.e., $\left(a_{i j}\right)=$ $\left(\xi_{i j}\right)\left(\eta_{i j}\right)^{*}$. Thus $\left\|\left(a_{i j}\right)\right\|_{A\left(\Gamma_{n}\right)} \leq 1$, by Haagerup's corollary. Let us verify that $\left(a_{i j}\right)=\left(\xi_{i j}\right)\left(\eta_{i j}\right)^{*}$ and $a_{i j}=\left(\xi_{i} \mid \eta_{j}\right)$. Recall that

$$
\begin{aligned}
a_{i j} & =a\left(g_{i}^{-1} g_{j}\right)=\xi * \tilde{\eta}\left(g_{i}^{-1} g_{j}\right)=\sum_{x \in G} \xi(x) \tilde{\eta}\left(x^{-1} g_{i}^{-1} g_{j}\right) \\
& =\sum_{x \in G} \xi(x) \bar{\eta}\left(g_{j}^{-1} g_{i} x\right)=\sum_{x \in G} \xi\left(g_{i}^{-1} x\right) \bar{\eta}\left(g_{j}^{-1} x\right)=\left(\xi_{i} \mid \eta_{j}\right) .
\end{aligned}
$$

If we write $\sum_{x \in G} \xi\left(g_{i}^{-1} x\right) \bar{\eta}\left(g_{j}^{-1} x\right)$ as $\sum_{k=1}^{n} \xi\left(g_{i}^{-1} g_{k}\right) \bar{\eta}\left(g_{j}^{-1} g_{k}\right)$ we see that $\left(a_{i j}\right)=\left(\xi_{i j}\right)\left(\eta_{i j}\right)^{*}$.

Now let us observe that

$$
\left\|\left(a_{i j}\right)\right\|_{A\left(\Gamma_{n}\right)}=\sup \left\{\left\|\left(a_{i j}\right) \circ\left(x_{i j}\right)\right\|_{M_{n}}:\left\|\left(x_{i j}\right)\right\|_{M_{n}} \leq 1\right\}
$$

where $\left(x_{i j}\right)$ is an arbitrary element with norm 1 or less in $M_{n}$ viewed as a $C^{*}$-algebra. In addition

$$
\left\|\left(a_{i j}\right)\right\|_{A(G)}=\sup \left\{\left\|\left(a_{i j}\right) \circ\left(x_{i j}\right)\right\|_{C^{*}(G)}:\left\|\left(x_{i j}\right)\right\|_{C^{*}(G)} \leq 1\right\}
$$

where $\left(x_{i j}\right)$ is an arbitrary element of norm one or less in $C^{*}(G)$ viewed as an (isometric) $C^{*}$-subalgebra of $M_{n}$ via the appropriate global Cayley map. We clearly have then that $\left\|\left(a_{i j}\right)\right\|_{A\left(\Gamma_{n}\right)} \geq\|a\|_{A(G)}$ and hence $\left\|\left(a_{i j}\right)\right\|_{A\left(\Gamma_{n}\right)}=\|a\|_{A(G)}$.

Finally, if $n>1$, then $\|I\|_{\mathrm{Tr}}=n>1=\|I\|_{A\left(\Gamma_{n}\right)}$ where $I$ is the $n \times n$ identity matrix. This shows that $A(G)$ is not isometrically a subalgebra of $\left(M_{n}, \circ,\|\cdot\|_{\mathrm{Tr}}\right)$, and we are done.

REMARK. We are dealing with a fundamental difference between the "dual space" and "completely bounded operator" points of view. $\left(M_{n}, \circ,\|\cdot\|_{\mathrm{Tr}}\right)$ is a Banach algebra, but it fails to be a Banach algebra for $n>1$ if the trace is normalized to 1 on $I$. When $n$ is infinite, renormalization is not even possible.

3. The infinite, discrete case. Using the completely bounded operator approach we now turn to defining the Fourier and Fourier-Stieltjes algebras for $\Gamma_{n}$ when $n$ is countably infinite. Here, the dual space approach apparently breaks down totally when one attempts to define the Fourier-Stieltjes algebra, $B\left(\Gamma_{\infty}\right)$, where $\Gamma_{\infty}$ is the principal transitive groupoid on, say, the natural numbers.

Now we can realize $\Gamma_{\infty}$ in much the same way as $\Gamma_{n}$ for $n$ finite, viz., $\Gamma_{\infty}=\left\{e_{i j}: 1 \leq i, j<\infty\right\}$, the matrix units for the (countably) infinite, complex matrices. 
Now the set of all uniformly bounded, complex functions on $\Gamma_{\infty}$, denoted $l^{\infty}\left(\Gamma_{\infty}\right)$, is none other than the (countably) infinite complex matrices with uniformly bounded entries, i.e., $l^{\infty}\left(\Gamma_{\infty}\right)=\left\{\left(x_{i j}\right)\right.$ : $\left.\sup _{i, j}\left|x_{i j}\right|<\infty\right\}$. If $\mathbf{N}$ denotes the natural numbers, and $l^{2}(\mathbf{N})$, the usual Hilbert space of (absolutely) square summable complex sequences, then not every element in $l^{\infty}\left(\Gamma_{\infty}\right)$ represents a bounded operator on $l^{2}(\mathbf{N})$.

For example, the matrix with every entry equal to 1 does not yield a bounded operator on $l^{2}(\mathbf{N})$, but it is in $l^{\infty}\left(\Gamma_{\infty}\right)$. Also it is an example of what we shall call a positive definite function on $\Gamma_{\infty}$.

Definition. A bounded function $f: \Gamma_{\infty} \rightarrow \mathbf{C}$ is positive definite if given any sequence of complex numbers $\lambda_{1}, \lambda_{2}, \lambda_{3}, \ldots$ which is 0 except for (at most) finitely many subscripts we have $\sum_{i, j=1}^{\infty} f\left(e_{i j}\right) \lambda_{i} \bar{\lambda}_{j} \geq 0$. The set of all (bounded) positive definite functions on $\Gamma_{\infty}$ will be denoted $P\left(\Gamma_{\infty}\right)$.

Proposition 3. The positive definite functions, $P\left(\Gamma_{\infty}\right)$, form a semigroup under pointwise multiplication.

Proof. This result, in other words, says that the Schur-Hadamard product of two (countably) infinite positive definite matrices is positive definite. This result follows from the finite case which is well known, cf. [1, Lemma 3.2].

Definition. The Fourier-Stieltjes algebra of $\Gamma_{\infty}$, denoted $B\left(\Gamma_{\infty}\right)$, as an algebra is the set of finite linear combinations of functions in $P\left(\Gamma_{\infty}\right)$.

REMARK. The finite linear combinations of elements from a semigroup always form an algebra by "abstract reasoning".

Let $\mathscr{C}\left(l^{2}(\mathbf{N})\right)$ be the $C^{*}$-algebra of compact operators on Hilbert space $l^{2}(\mathbf{N})$. To simplify notation let $\mathscr{C}=\mathscr{C}\left(l^{2}(\mathbf{N})\right)$ where convenient.

Proposition 4. The set of all completely bounded maps of $\mathscr{C}$ into itself, i.e., $\mathscr{D}(\mathscr{C})$, contains $B\left(\Gamma_{\infty}\right)$ via the Schur-Hadamard "action" of $B\left(\Gamma_{\infty}\right)$ on $\mathscr{C}\left(l^{2}(\mathbf{N})\right)$.

Proof. If $p=\left(p_{i j}\right)$ is in $P\left(\Gamma_{\infty}\right)$, it defines a map of $\mathscr{C}\left(l^{2}(\mathbf{N})\right)$ into itself by pointwise multiplication, i.e., Schur-Hadamard product. If $\left(x_{i j}\right) \in \mathscr{C}\left(l^{2}(\mathbf{N})\right)$, then $p \circ x=\left(p_{i j}\right) \circ\left(x_{i j}\right)=\left(p_{i j} x_{i j}\right)$. In fact the map $x \in \mathscr{C} \mapsto p \circ x \in \mathscr{C}$ is completely positive and has a completely 
bounded norm equal to $\sup _{i} p_{i i}$. Since $b \in B\left(\Gamma_{\infty}\right)$ means $b$ is a linear combination of positive definite functions on $\Gamma_{\infty}, x \in \mathscr{C} \mapsto b \circ x \in \mathscr{C}$ is a linear combination of completely positive operators, hence $x \mapsto b \circ x$ is a completely bounded map.

Thus $B\left(\Gamma_{\infty}\right)$ identifies with a normed subalgebra of $\mathscr{D}(\mathscr{C})$. Now if $f \in l^{\infty}\left(\Gamma_{\infty}\right)$ we can consider the pointwise or Schur-Hadamard product, $f \circ x$, for $x \in \mathscr{C}$. We can ask the question: For which $f \in l^{\infty}\left(\Gamma_{\infty}\right)$ is $x \in \mathscr{C} \mapsto f \circ x \in \mathscr{C}$ a (completely) bounded map? The answer is:

THEOREM 2. The Fourier-Stieltjes algebra $B\left(\Gamma_{\infty}\right)$ consists exactly of those $f \in l^{\infty}\left(\Gamma_{\infty}\right)$ for which the map $x \in \mathscr{C} \mapsto f \circ x \in \mathscr{C}$ is completely bounded.

Proof. Returning back to Haagerup's theorem in the first section, let $\Omega=\mathbf{N}$ and identify $\Gamma_{\infty}$ with $\mathbf{N} \times \mathbf{N}$ via the correspondence $e_{i j} \Leftrightarrow$ $(i, j)$ for all $i, j \in \mathbf{N}$. Let $\mu$ be the counting measure on $\mathbf{N}$. Then $H=L^{2}(\Omega, \mu)=l^{2}(\mathbf{N}), L^{\infty}(\Omega \times \Omega, \mu \times \mu)=l^{\infty}\left(\Gamma_{\infty}\right)$.

Now if $f \in l^{\infty}\left(\Gamma_{\infty}\right)$ the map $x \in \mathscr{C} \mapsto f \circ x \in \mathscr{C}$ has norm one or less if and only if the double transpose of this map, i.e., $x \in \mathscr{B}\left(l^{2}(\mathbf{N})\right) \mapsto$ $f \circ x \in \mathscr{B}\left(l^{2}(\mathbf{N})\right)$, (is $\sigma$-weakly continuous and) has norm one or less. This latter condition is condition (2) of Haagerup's theorem.

Now we can without loss of generality assume $f=f^{*}$, where $f^{*}\left(e_{i j}\right)=\overline{f\left(e_{j i}\right)}$, by the following. We have

$$
f=\left(\frac{f+f^{*}}{2}\right)+i\left(\frac{f-f^{*}}{2 i}\right)
$$

and

$$
\left\|\frac{f+f^{*}}{2}\right\|_{\mathscr{D}(\mathscr{C})} \leq 1 \text { and } \quad\left\|\frac{f-f^{*}}{2 i}\right\|_{\mathscr{D}(\mathscr{C})} \leq 1 .
$$

So if $f=f^{*}$ and $f \in l^{\infty}\left(\Gamma_{\infty}\right)$ is such that $x \in \mathscr{C} \mapsto f \circ x \in \mathscr{C}$ has (completely) bounded norm of one or less, then Haagerup's theorem tells us that there is a separable Hilbert space $\mathscr{H}$ and two sequences of vectors in $\mathscr{H}$, viz., $\xi_{1}, \xi_{2}, \ldots$ and $\eta_{1}, \eta_{2}, \ldots$, such that $\sup _{i}\left\|\xi_{i}\right\| \leq$ $1, \sup _{j}\left\|\eta_{j}\right\| \leq 1$ and $f\left(e_{i j}\right)=\left(\xi_{i} \mid \eta_{j}\right)$ for all $i$ and $j$.

Let $p$ and $q$ be elements in $P\left(\Gamma_{\infty}\right)$ defined by $p\left(e_{i j}\right)=\left(\xi_{i} \mid \xi_{j}\right), q\left(e_{i j}\right)$ $=\left(\eta_{i} \mid \eta_{j}\right)$ for all $i$ and $j$. The matrix

$$
\left(\begin{array}{ll}
p & f \\
f & q
\end{array}\right)
$$


is positive definite in the appropriate sense, and it follows that $(p+q) / 2 \pm f \in P\left(\Gamma_{\infty}\right)$. Thus

$$
2 f=\left(\frac{p+q}{2}+f\right)-\left(\frac{p+q}{2}-f\right),
$$

and hence $f$ is a linear combination of elements from $P\left(\Gamma_{\infty}\right)$. Thus $f \in B\left(\Gamma_{\infty}\right)$.

COROllary. The Fourier-Stieltjes algebra, $B\left(\Gamma_{\infty}\right)$, is a Banach algebra, viz., $B\left(\Gamma_{\infty}\right)$ is a closed subalgebra of $\mathscr{D}(\mathscr{C})$.

REMARK. We here give $B\left(\Gamma_{\infty}\right)$ the normed algebra structure it inherits "naturally" as an algebra of completely bounded operators.

Proof. $\mathscr{D}(\mathscr{C})$ is a Banach algebra, cf. [13, Proposition 1]. Consider $B\left(\Gamma_{\infty}\right) \subset \mathscr{D}(\mathscr{C})$ as in Proposition 4. Let $\left\{b_{n}\right\} \subset B\left(\Gamma_{\infty}\right)$ be a sequence and let $\left\|b_{n}-b\right\|_{\mathscr{D}(\mathscr{C})} \rightarrow 0, b \in \mathscr{D}(\mathscr{C})$. Let $e_{i j}$, for fixed $i$ and $j$, be simultaneously considered as an element of $\Gamma_{\infty}$ and of $\mathscr{C}$. Now

$$
\left\|b_{n}\left(e_{i j}\right) e_{i j}-b\left(e_{i j}\right)\right\|_{\mathscr{C}}=\left\|\left(b_{n}-b\right)\left(e_{i j}\right)\right\|_{\mathscr{C}} \leq\left\|b_{n}-b\right\|_{\mathscr{D}(\mathscr{C})} .
$$

Thus $b\left(e_{i j}\right)=\lim _{n \rightarrow \infty} b_{n}\left(e_{i j}\right) e_{i j}=\lambda_{i j} e_{i j}$ for a suitable $\lambda_{i j} \in \mathbf{C}$. Thus the operator $b \in \mathscr{D}(\mathscr{C})$ is none other than Schur-Hadamard multiplication by the matrix $\left(\lambda_{i j}\right) \in l^{\infty}\left(\Gamma_{\infty}\right)$. Thus by Theorem $2, b \in B\left(\Gamma_{\infty}\right)$. Thus $B\left(\Gamma_{\infty}\right)$ is complete, i.e., it is a Banach algebra.

In analogy with the group case we can define $A\left(\Gamma_{\infty}\right)$ to be the closure in $B\left(\Gamma_{\infty}\right)$ of the functions with finite support.

Now let $G$ be a countably infinite discrete group, and enumerate the elements of $G$ as $g_{1}, g_{2}, g_{3}, \ldots$ As in the case of a finite group in $\S 2$, we can construct corresponding Cayley representations for $G$ and $B(G)$. We can show that $B\left(\Gamma_{\infty}\right)$ not only contains (many copies of) $B(G)$ but all the "completely bounded multipliers" of $A(G)$ as well (inclusions are perhaps not isometric). We have already seen that for finite $n$ $A\left(\Gamma_{n}\right)$ contains (usually several copies of) the Fourier algebra of any group of order $n$. Clearly $A\left(\Gamma_{m}\right)$ contains $A\left(\Gamma_{n}\right)$ for $n \leq m$. It follows that in a sense we shall not make precise that $B\left(\Gamma_{\infty}\right)$ "contains" the dual algebras of all countable (both finite and infinite) groups. This is the beginning of a much larger story, however. We hope to return and tell more of this story in subsequent papers. The main point of this paper has been to show that without adopting the "completely bounded operator" approach an interesting investigation into noncommutative harmonic analysis cannot take place. 
Acknowledgment. The author wishes to acknowledge numerous conversations about the content of this paper with Magnus B. Landstad. Also this paper would not have been written were it not for the generous hospitality of Magnus B. Landstad during the summer of 1987.

\section{REFERENCES}

[1] C. Berg, and G. Forst, Potential Theory on Locally Compact Groups, SpringerVerlag, Berlin, Heidelberg, New York, 1975.

[2] J. Dixmier, Les $C^{*}$-algèbres et leurs Représentations, Cahiers Scientifiques, Fasc. 29, Gauthier-Villars, Paris, 1964.

[3] P. Eymard, L'algèbre de Fourier d'un groupe localement compact, Bull. Soc. Math. France, 92 (1964), 181-236.

[4] U. Haagerup, Decomposition of completely bounded maps on operator algebras, handwritten notes, September 1980.

[5] E. Hewitt and K. Ross, Abstract Harmonic Analysis, Vol. 1, Springer-Verlag, Berlin, 1963.

[6] Abstract Harmonic Analysis, Vol. 2, Springer-Verlag, Berlin, 1970.

[7] V. I. Paulsen, Completely Bounded Maps and Dilations, Pitman Research Notes in Mathematics Series 146, John Wiley and Sons, Inc., New York.

[8] V. I. Paulsen, and S. C. Power, Schur Products and Matrix Completions, preprint.

[9] W. Rudin, Fourier Analysis on Groups, Interscience, New York, 1962.

[10] M. Takesaki, Theory of Operator Algebras, I, Springer-Verlag, Berlin-HeidelbergNew York, 1979.

[11] M. E. Walter, $W^{*}$-algebras and nonabelian harmonic analysis, J. Funct. Anal., 11 (1972), 17-38.

[12] _ A duality between locally compact groups and certain Banach algebras, J. Funct. Anal., 17 (1974), 131-160.

[13] _ Dual algebras, Math. Scand., 58 (1986), 77-104.

Received February 25, 1988. This research was supported by a travel grant from the Norwegian Marshall Fund.

UNIVERSITY OF COLORADO

BOULDER, CO 80309-0426 



\section{PACIFIC JOURNAL OF MATHEMATICS EDITORS}

\author{
V. S. VARADARAJAN \\ (Managing Editor) \\ University of California \\ Los Angeles, CA 90024 \\ Herbert Clemens \\ University of Utah \\ Salt Lake City, UT 84112 \\ THOMAS ENRIGHT \\ University of California, San Diego \\ La Jolla, CA 92093
}

R. FINN

Stanford University

Stanford, CA 94305

HeRmanN FlaschKa

University of Arizona

Tucson, AZ 85721

VAUGHAN F. R. JONES

University of California

Berkeley, CA 94720

STEVEN KeRCKHOFF

Stanford University

Stanford, CA 94305
RoBION KIRBY

University of California

Berkeley, CA 94720

C. C. MOore

University of California

Berkeley, CA 94720

HAROLD STARK

University of California, San La Jolla, CA 92093

\section{ASSOCIATE EDITORS}
R. ARENS
E. F. BECKENBACH
B. H. NeUmanN
F. WolF
K. YosHIDA (1906-1982)

\section{SUPPORTING INSTITUTIONS}
UNIVERSITY OF ARIZONA
UNIVERSITY OF BRITISH COLUMBIA CALIFORNIA INSTITUTE OF TECHNOLOGY UNIVERSITY OF CALIFORNIA MONTANA STATE UNIVERSITY UNIVERSITY OF NEVADA, RENO NEW MEXICO STATE UNIVERSITY OREGON STATE UNIVERSITY
UNIVERSITY OF OREGON UNIVERSITY OF SOUTHERN CALIFORNIA STANFORD UNIVERSITY UNIVERSITY OF HAWAII UNIVERSITY OF TOKYO UNIVERSITY OF UTAH WASHINGTON STATE UNIVERSITY UNIVERSITY OF WASHINGTON 


\section{Pacific Journal of Mathematics}

\section{Vol. 137, No. $1 \quad$ January, 1989}

V. S. Varadarajan, Henry Abel Dye $\ldots \ldots \ldots \ldots \ldots \ldots \ldots \ldots \ldots \ldots$ iii

Huzihiro Araki, An application of Dye's theorem on projection lattices to orthogonally decomposable isomorphisms $\ldots \ldots \ldots \ldots \ldots \ldots \ldots \ldots \ldots$

Richard Arens, The limit of a sequence of squares in an algebra need not be

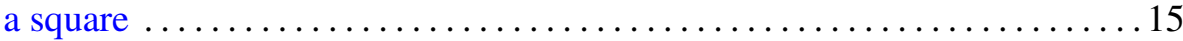

William Arveson, An addition formula for the index of semigroups of

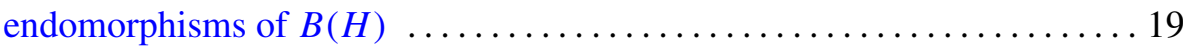

Robert James Blattner and Susan Montgomery, Crossed products and

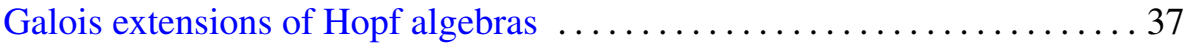

Erik Christensen and Allan M. Sinclair, On the vanishing of $H^{n}\left(\mathscr{A}, \mathscr{A}^{*}\right)$

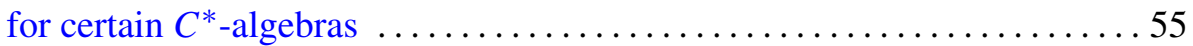

Philip C. Curtis, Jr. and Michael M. Neumann, Nonanalytic functional

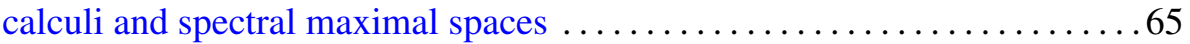

George A. Elliott and David E. Handelman, Addition of $C^{*}$-algebra extensions

Yaakov Friedman and Bernard Russo, Some affine geometric aspects of

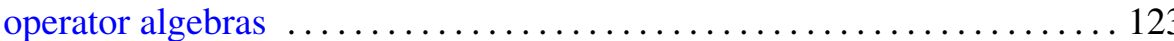

Valentin Ya. Golodets and Sergey D. Sinelshchikov, Regularization of actions of groups and groupoids on measured equivalence relations . . . 145

Irving Kaplansky, CCR-rings 155

Hideki Kosaki, Characterization of crossed product (properly infinite case)

Gert Kjærgaard Pedersen, Three quavers on unitary elements in $C^{*}$-algebras

Sorin Popa, Relative dimension, towers of projections and commuting squares of subfactors

Martin E. Walter, On a new method for defining the norm of

Fourier-Stieltjes algebras 